\title{
Rheumatic heart disease: current status of diagnosis and therapy
}

\author{
Ferande Peters ${ }^{1}$, Ganesan Karthikeyan ${ }^{2}$, Jessica Abrams $^{3}$, Lorrein Muhwava ${ }^{3}$, Liesl Zühlke ${ }^{3,4}$ \\ ${ }^{1}$ Cardiovascular pathophysiology and Genomic Unit, University of the Witwatersrand, Johannesburg, South Africa; ${ }^{2}$ Cardiothoracic Sciences Centre, \\ All India Institute of Medical Sciences, New Delhi, India; ${ }^{3}$ Division of Paediatric Cardiology, Department of Paediatrics, Red Cross Children's \\ Hospital, University of Cape Town, Cape Town, South Africa; ${ }^{4}$ Division of Cardiology, Department of Medicine, Groote Schuur Hospital, \\ University of Cape Town, Cape Town, South Africa \\ Contributions: (I) Conception and design: L Zühlke; (II) Administrative support: J Abrams, L Muhwava; (III) Provision of study materials or patients: \\ F Peters; (IV) Collection and assembly of data: L Zühlke, J Abrams, L Muhwava; (V) Data analysis and interpretation: All authors; (VI) Manuscript \\ writing: All authors; (VII) Final approval of manuscript: All authors. \\ Correspondence to: Professor Liesl Zühlke. 2.17 Institute of Child Health, Red Cross Children's Hospital, Klipfontein Rd Rondebosch 7700, Cape \\ Town, South Africa. Email: Liesl.zuhlke@uct.ac.za.
}

\begin{abstract}
Rheumatic heart disease (RHD) is the only preventable cardiovascular disease which causes significant morbidity and mortality particularly in low- and middle-income countries. Early clinical diagnosis is key, the updated Jones criteria increases the likelihood of diagnosis in endemic settings, including the echo diagnosis of sub-clinical carditis, polyarthralgia and monoarthritis as well as amended thresholds of minor criteria. The mainstay of rheumatic heart valve disease (RHVD) is a thorough clinical and echocardiographic investigation while severe disease is managed with medical, interventional and surgical treatment. In this report we detail some of the more recent epidemiological findings and focus on the diagnostic and interventional elements of the specific valve lesions. Finally, we discuss some of the recent efforts to improve medical and surgical management for this disease. As we are already more than a year from the historic 2018 World Heart Organization Resolution against Rheumatic Fever and Rheumatic Heart Disease, we advocate strongly for renewed efforts to prioritize this disease across the endemic regions of the world.
\end{abstract}

Keywords: Echocardiography; Jones criteria; rheumatic heart disease (RHD); surgery; World Health Organization Resolution

Submitted Sep 16, 2019. Accepted for publication Oct 28, 2019.

doi: $10.21037 / \mathrm{cdt} .2019 .10 .07$

View this article at: http://dx.doi.org/10.21037/cdt.2019.10.07

\section{Introduction}

Rheumatic heart disease (RHD) is a chronic heart valve condition affecting up to 40 million people worldwide, predominantly poverty-stricken children and young adults. RHD is caused by a preventable infection of the bacterium Group A Streptococcus (Strep A) which may lead to an autoimmune response in the body, namely acute rheumatic fever (ARF). If Strep A and ARF go untreated, repeated infections are more likely to occur. While fever and other rheumatic symptoms often resolve, ARF-associated carditis can result in permanent damage to at least one of the four heart valves. Long-term consequences of RHD may include stroke, heart failure, and premature mortality (1).
While the global incidence of ARF and RHD has declined, it remains endemic in regions which experience barriers to healthcare as well as crowded living environments (2). These factors enable the spread of Strep A and development of RHD, causing half a million deaths annually (3). In high-income countries RHD is more frequently being identified among Indigenous and migrant populations, with $87 \%$ of RHD patients in Australia belonging to Aboriginal and Torres Strait Islander people (4).

There has been a renewed interest in addressing RHD and its precursors following the release of revised diagnostic guidelines, updated global burden of disease estimates, and resolutions by various international agencies $(5,6)$. Most recently, the $71^{\text {st }}$ World Health Assembly 
approved a World Health Organization Resolution on Rheumatic Fever and Rheumatic Heart Disease (7). A number of recommendations are specified for combating the burden of disease including national RHD programme implementation, improving prevention, diagnosis, and treatment of Strep A and ARF, and ensuring a consistent supply of BPG in primary care facilities.

In this review we discuss the genesis of RHD, its diagnosis and treatment, and future directions for the disease given its new position on the international agenda.

\section{Burden of disease}

The Global Burden of Disease estimates for RHD have described a variable pattern with the overall estimates for disease aged-standardized death rate decreasing overall by as much as $47.8 \%$ in the period 1990-2015 (3). However, the difference in endemic versus non-endemic rates remains vast with 3.4/100,000 in non-endemic regions of the world compared to $444 / 100,000$ in endemic countries. The regions at highest risk are those in Oceania, South Asia and Central Sub-Saharan Africa with the highest rates of agestandardized death rates, prevalence and disability adjusted life years (DALYS) and the highest health-related burden of RHD in these regions and surrounds (8). It is clear that the burden of RHD although attenuated in high-income and some middle-income countries, continue unabated in these regions with untold suffering and deaths per year.

\section{Diagnosis of ARF}

ARF usually occurs 2-3 weeks following a Strep A throat infection. The organs affected include the brain, skin, joints and heart. The most common clinical manifestations of $\mathrm{ARF}$ are carditis and arthritis, followed by chorea (a delayed manifestation), subcutaneous nodules, and erythema marginatum, which are described in detail in the paragraphs below.

The clinical presentation of ARF is characterized by fever which usually occurs 2 to 3 weeks after an episode of pharyngitis. The clinical features associated with joint involvement vary from arthralgia to polyarthritis. The arthritis most commonly afflicts large joints such as the wrists, elbows, knees and ankles and can be migratory in its presentation. It is often initially asymmetric, beginning in the lower limbs, and usually involves other joints once the inflammation of the initial joints involved have subsided. The hallmark of arthritis related to ARF is its prompt improvement is response to the use of non-steroidal anti- inflammatory drugs.

The sine qua non for the diagnosis of carditis is valvulitis which presents as mitral regurgitation (MR) or less commonly aortic regurgitation (AR). Myocarditis, pericarditis and less commonly heart block are other features of carditis. In some instances, patients may present with heart failure which usually is related to severe valvular regurgitation and surgical intervention maybe required if medical therapy fails.

The chorea observed with ARF occurs more frequently in women and occurs later in the course of ARF. Consequently, evidence of a prior Strep A infection is not found and exclusion of other causes of chorea together with echocardiographic evidence of carditis is often required to confirm the diagnosis. The presence of subcutaneous nodules maybe detected on the extensive surfaces of the arms and legs as well as the head. These nodules are usually less than $2 \mathrm{~cm}$, mobile and painless and thus easily missed if not actively searched for on clinical examination. Erythema marginatum is an uncommon transient manifestation and usually is detected as an erythematosus rash which has a pale center and is usually found on the back. This rash may occur early in the course of the disease and may be more frequently detected with concomitant carditis.

In 2015 the revised Jones criteria were published and remains the current recommended criteria to be utilized when establishing a diagnosis of ARF (9). Similar to prior iterations of the Jones criteria, proof of a recent Strep A infection is required together with either 2 major or 1 major and 2 minor criteria. Evidence of a preceding Strep A infection include an elevated or rising anti-streptolysin-O or other streptococcal antibody, or a positive throat culture, or rapid antigen test for Strep A, or recent scarlet fever. Caution is advised in terms of normal values of these antibodies in different populations, as well as the sensitivity and specificity of rapid antigen tests for Strep A (10). The two major changes in the 2015 revised criteria relate to the utilization of different criteria for diagnosis of individuals from different populations and secondly the utilization of echocardiography for the diagnosis of carditis.

The first step in assessing a patient with possible ARF is to determine whether they are from a low risk population or a moderate/high risk population. Low risk is identified by populations where the incidence of ARF in children aged $5-14$ years old is less than $2 / 100,000 /$ year or a prevalence of RHD less than or equal to $1 / 1,000$ population/year. Patients are assigned to the medium/high risk category if they are not low risk. The aim of this risk stratification is to improve the diagnostic pickup rate in high risk/endemic 
Table 1 The 2012 World Heart Federation criteria for the diagnosis of rheumatic heart disease (11)

\begin{tabular}{|c|c|}
\hline Sub-clinical RHD & Doppler criteria-all four to be satisfied \\
\hline \multicolumn{2}{|l|}{$\begin{array}{l}\text { Pathological } \\
\text { regurgitation lesion }\end{array}$} \\
\hline \multirow[t]{4}{*}{ Mitral regurgitation } & Visualised in $\geq 2$ views \\
\hline & Color jet length $\geq 2 \mathrm{~cm}$ \\
\hline & Peak CW jet velocity $>3 \mathrm{~m} / \mathrm{s}$ \\
\hline & Pansystolic jet \\
\hline \multirow[t]{4}{*}{ Aortic regurgitation } & Visualised in $\geq 2$ views \\
\hline & Color jet length $\geq 1 \mathrm{~cm}$ \\
\hline & Peak CW jet velocity $>3 \mathrm{~m} / \mathrm{s}$ \\
\hline & Pandiastolic jet \\
\hline \multicolumn{2}{|l|}{ Morphological criteria } \\
\hline \multirow[t]{4}{*}{ Mitral } & Valve thickening $>3 \mathrm{~mm}$ \\
\hline & Chordal abnormality \\
\hline & Restricted posterior leaflet \\
\hline & Excessive systolic motion of leaflet tip \\
\hline \multirow[t]{4}{*}{ Aortic } & Leaflet thickening/irregularity \\
\hline & Coaptation defect \\
\hline & Restricted leaflet motion \\
\hline & Prolapse \\
\hline
\end{tabular}

Definite RHD: pathological MR/AR with 2 or more respective morphological criteria, mitral stenosis with mean gradient $>4$ $\mathrm{mmHg}$ or borderline RHD of both the mitral and aortic valve. Borderline RHD: at least two morphological features of RHD of the MV without pathological MR or MS or pathological AR/ MR with no morphological criteria. RHD, rheumatic heart disease; MR, mitral regurgitation; $\mathrm{AR}$, aortic regurgitation; $\mathrm{CW}$, continuous wave Doppler.

areas. The clinical utility of a diagnostic test is influenced by pretest probability and background disease prevalence, a single set of diagnostic criteria may no longer be sufficient for all population groups and in all geographic regions. New criteria to be applied to patients from a medium /high risk area include either polyarthralgia or mono arthritis in addition to polyarthritis as a major criterion and minor criteria which include a fever less than 38 degrees Celsius and the presence of Monoarthralgia.

\section{Diagnosis of carditis}

Echocardiography is the gold standard for the diagnosis of carditis. The major manifestation of carditis is valvulitis which manifests most commonly as mitral and aortic incompetence. From a practical point of view, a diagnosis of carditis should be avoided if no evidence of valvular dysfunction is found (9). In the revised Jones criteria of 2015, carditis may be clinical or subclinical. Subclinical carditis refers to the detection of echocardiographic valvular abnormalities consistent with ARF in the absence of signs of valvular dysfunction on standard auscultation.

The key to the diagnosis of valvular dysfunction requires integration of typical morphological abnormality with evidence of pathological valvular regurgitation. The criteria for pathological regurgitation are similar to that used in the World Heart Federation (WHF) criteria (Table 1) and was first described in the late nineties as a discriminator for silent carditis in ARF (12). The mitral morphological features suggestive of ARF are annular dilatation, beading and focal thickening of the leaflet, prolapse of the anterior mitral leaflet, elongated chordae and ruptured chordae (9). Importantly, no features of chronic RHD should be found. The morphological aortic criteria proposed as similar to that used in the diagnosis of chronic rheumatic aortic disease (Table 1).

Echocardiography can also be utilized to identify pericardial effusion as well as focal or global left ventricular dysfunction which are other supportive manifestations of carditis. Perhaps the greatest role of echocardiography is to identify alternate diagnoses such as congenital heart disease, mitral valve prolapses or to exclude physiological murmurs which may be misinterpreted by clinical evaluation as evidence of carditis. Recurrent carditis may be diagnosed in known patients with either prior ARF or who present with echocardiographic signs of chronic RHD and have evidence of recent Strep A infection and display other features of ARF (9). It is important to note that ARF can be an extremely severe condition and even lead to death at the first presentation; of concern in endemic countries is an anecdotal impression of more severe presentations of ARF, requiring surgery and leading to mortality (13).

\section{Chronic rheumatic heart valve disease (RHVD)}

Chronic RHVD commonly manifests with symptomatic valvular disease with heart failure, atrial fibrillation (AF) and pulmonary hypertension (PHT) being the predominant clinical manifestations $(14,15)$. A history of preceding $\mathrm{ARF}$ is uncommon and the mitral valve is most commonly affected followed by the aortic valve, tricuspid valve (TV) 

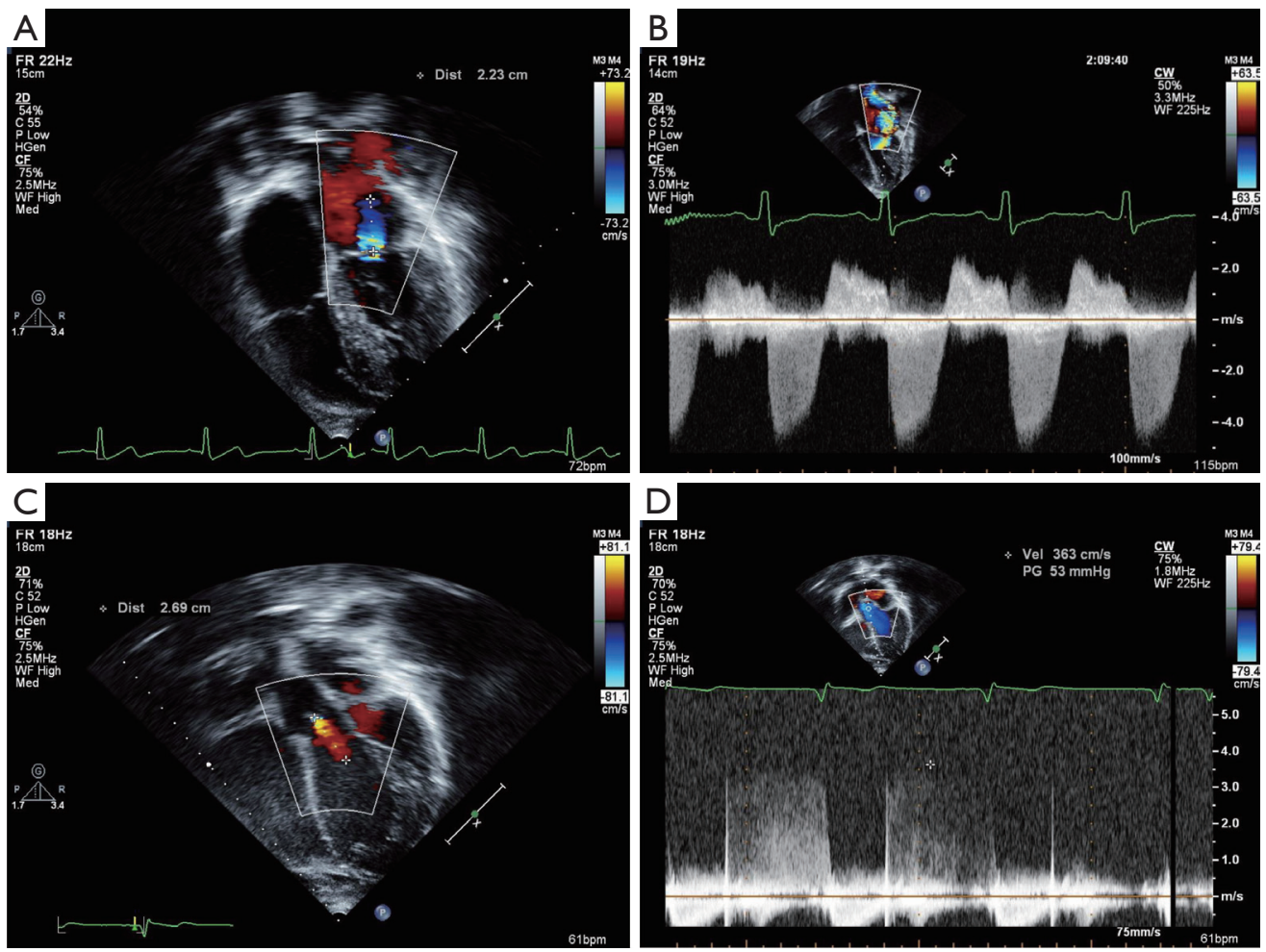

Figure 1 WHF criteria: (A,B) mitral valve and (C,D) aortic valve. WHF, World Heart Federation.

and very rarely the pulmonary valve. The involvement of hemodynamically significant rheumatic pulmonary valve disease is extremely uncommon and echocardiographic evaluation of the pulmonary valve in clinical practice more commonly will identify functional pulmonary regurgitation secondary to PHT.

Contemporary data from the Global Rheumatic Heart Disease Registry (REMEDY) revealed that the most common valve lesions encountered were mixed left sided lesions with multivalvular involvement occurred in almost two-thirds of subjects (15). The assessment of these often-challenging clinical scenarios should be based on standard echocardiographic guidelines to assess the regurgitant or stenotic lesion encountered. These findings should be correlated with surrogates of left ventricular function, right ventricular function and PHT. Integration of clinical intuition, echocardiographic data and invasive hemodynamic assessment may occasionally be required to determine the significance of mixed and or multi valvular disease in selected cases.

The utilization of screening echocardiography in various studies have revealed that the true burden of asymptomatic RHD is considerably greater than that detected clinically.
Most individuals have minor valvular dysfunction which may progress in severity or even regress $(11,16-19)$. Following considerable debate on how best to differentiate true RHD pathology detected on echocardiography from normality, the WHF criteria for the diagnosis of RHD proposed to aid diagnosis of these more subtle manifestations of subclinical RHD (Table 1 and Figure 1) (20). However, for a variety of socioeconomic and scientific reasons, the routine utilization of screening echocardiography is not recommended for current clinical practice. In clinical scenarios where echocardiography is performed and equivocal valve lesions are identified, the utilization of the WHF criteria often can aid in the differentiation of RHD from normality and assist in the diagnosis of subclinical carditis in the context of ARF. More recent prevalence estimates from Africa using the $2012 \mathrm{WHF}$ echocardiographic criteria range from $11.8 / 1,000$ in Zambia (21) to $19.0 / 1,000$ in Ethiopia (22), and 34.0/1,000 in Malawi $(23,24)$.

\section{$M R$}

Isolated MR is more commonly encountered in individuals younger than 30 years of age (25). A key issue is to 


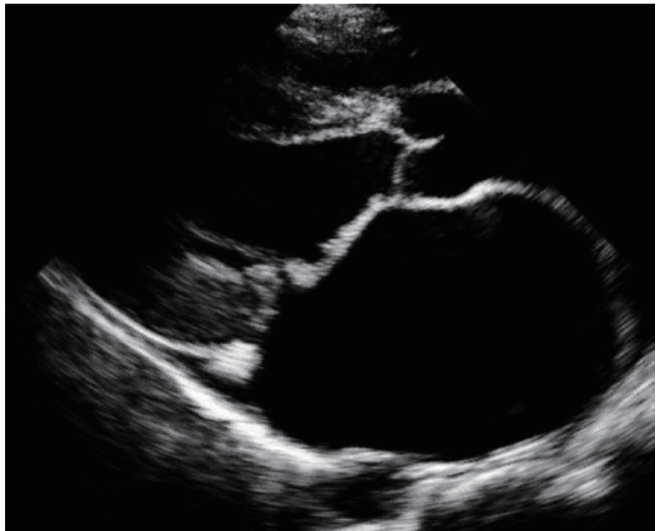

Figure 2 Restriction of posterior leaflet and failure of coaptation.

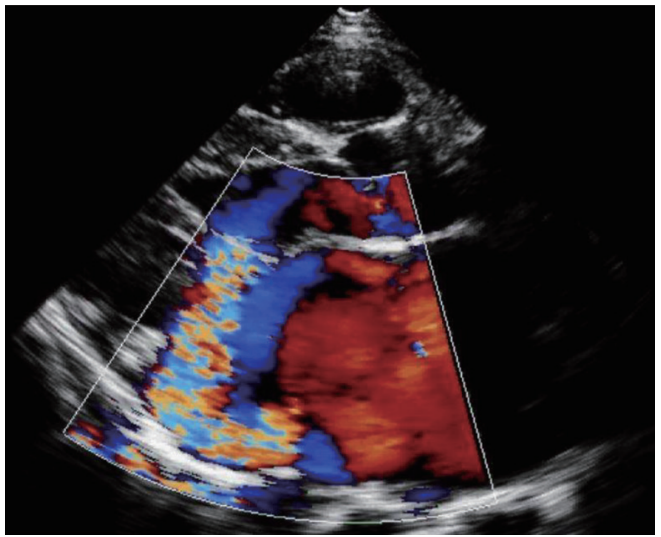

Figure 3 Eccentric mitral regurgitation jet.

evaluate the morphology of the valve to determine that the echocardiographic features are consistent with that of chronic RHVD. In addition to the functional and morphological abnormalities also described in the WHF criteria (Figure 2), other common features include doming of the anterior mitral leaflet and shortening of the anterior leaflet accompanied by varying degrees of leaflet thickening and calcification. The terms "dog-leg" or "hockey-stick" deformities address this combination of features, characteristic of chronic RHVD. The jet of MR is often eccentric and is due to malcoaptation and or shortening of the anterior leaflet with an immobile or restricted posterior leaflet most commonly having a Carpentier type 3 abnormality (Figure 3) (26).

The aforementioned morphological abnormalities contributing to the eccentric jet of MR are distinctly different to that observed in myxomatous mitral valve prolapse or in the appropriately aged individual with ARF. In ARF an eccentric MR jet is accompanied by prolapse of the anterior leaflet with focal beading/leaflet thickening, annular dilatation and choral elongation or rupture (20). These patients have no signs of chronic RHD. If an individual (usually less than 30 years of age) presents with either ARF with some echocardiographic signs of chronic RHD or alternatively has echocardiographic features of chronic RHD but has unexpected worsening of the MR or clinical features of possible ARF, recurrent ARF may need exclusion and should be based on the recommendations outlined in the revised Jones criteria of 2015 (9). Once a diagnosis of chronic rheumatic MR is made and its severity clarified, the LVESD, LVEF, pulmonary artery pressure, and presence and mechanism of TR must be carefully evaluated and correlated with the degree of MR severity.

\section{Mitral stenosis (MS)}

MS is the most common isolated lesion encountered after the age of 30 years $(14,26)$. It is also the most common valvular lesion that complicates the course of pregnancy imposing significant maternal and fetal morbidly and mortality (27-30). Unfortunately most cases of MS are detected when complications such as heart failure, AF, PHT, and embolic stroke already exist (31).

The key pathological feature of rheumatic MS is commissural fusion which can be detected on echocardiography by short axis imaging of the mitral valve and inferred by the presence of doming of the anterior leaflet during diastole. The posterior leaflet usually has restricted leaflet motion during diastole and is not infrequently immobile. The leaflets are thickened at their tips and may have superimposed calcification. There are varying degrees of thickening and calcification of the leaflet body which may be accompanied by varying degrees of chordal shortening and fusion. This variability in the rheumatic process forms the basis of the Wilkins score to assess the likelihood of a valve being suitable for percutaneous mitral commissurotomy (PMC).

In older individuals, calcification maybe observed in the commissures and is crucial to detect since this is associated with an unfavorable outcome if PMC is performed. In older individuals the absence of echocardiographic signs inferring commissural fusion as well the predilection of calcification and thickening of the basal portions of the leaflet usually infer non rheumatic MS. The assessment of the severity of MS should be based on accepted guidelines and performed in the first instance using echocardiography. Integration of direct planimetry of the mitral valve area $(2 \mathrm{D} / 3 \mathrm{D})$, the mean mitral 


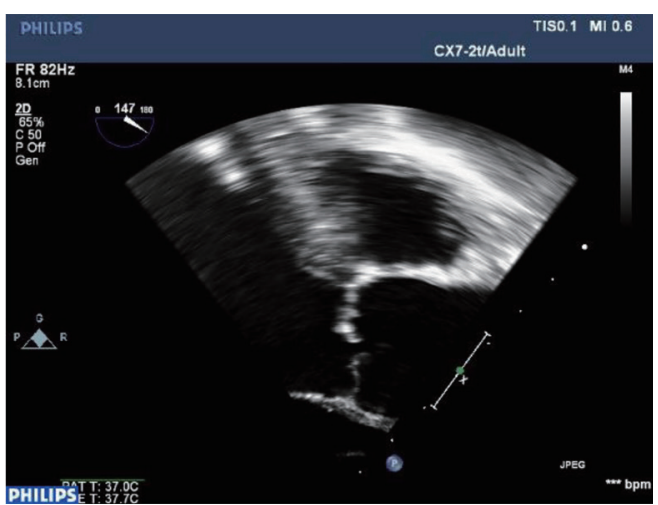

Figure 4 Beaded aortic valve doming in systole.

gradient and pulmonary artery pressure are the key elements of evaluation. The use of other Doppler based techniques to evaluate the mitral area is often used but maybe more challenging to quantify mitral valve area when concomitant valvular lesions such as moderate or severe $\mathrm{AR}$ are present.

\section{$A R$}

The morphology of the aortic valve on echocardiography reveals that the leaflets may be thickened with varying degrees of calcification often associated with restricted leaflet motion. Doming of the leaflets may be noted and on short axis commissural fusion may be observed. The consequence of these morphological changes is that the leaflets have a central coaptation defect during diastole and consequently a central jet of AR (Figure 1). The aforementioned morphological features may also be present in ARF except for the presence of calcification. There is often some degree of stenosis of the leaflets during systole but when the aortic valve area is greater than $2 \mathrm{~cm}^{2}$ the predominant hemodynamic effect is that of $\mathrm{AR}$ and volume overload of the left ventricle. The echocardiographic features of $\mathrm{LV}$ dilation and eccentric hypertrophy as well as the clinical features of a high output state are observed in moderate or severe AR. These characteristic hemodynamic changes maybe attenuated in patients with AR and MS. This clinical scenario may require judicious clinical and echocardiographic evaluation to identify the severity of the AR and MS which will consequently influence clinical decision making.

\section{Aortic stenosis (AS)}

Unlike degenerative AS, rheumatic AS is observed infrequently as an isolated lesion (Figure 4). More commonly it will be observed with coexisting AR or mitral valve disease (14). The morphology of the valve is similar to that observed in AR and can be distinguished from degenerative AS by its characteristic normal appearance of the basal portion of the leaflets. In contradistinction degenerative AS always afflicts the basal portion of the leaflets and does not cause commissural fusion. This distinctive difference is more challenging in older patients with RHD where degenerative changes may coexist. In the younger patient exclusion of congenital causes of AS must be performed by careful evaluation of the aortic valve in short axis views. This is particularly important in the patient with a bicuspid aortic valve where doming of the leaflets may be observed.

\section{TV disease}

Rheumatic TV pathology is usually found in association with left sided valvular heart disease. The TV should be evaluated from multiple views to visualize all three leaflets since not infrequently morphological abnormalities are more pronounced on some leaflets compared to others. This is often associated with leaflet thickening, doming and tethering accompanied by varying degrees of calcification causing more commonly tricuspid regurgitation (TR), followed by mixed TV disease and least commonly isolated tricuspid stenosis (TS). The presence of rheumatic morphological abnormality together with some degree of stenosis allows aides differentiation of rheumatic TR from functional TR. The tricuspid annulus should always be measured and ideally indexed to body surface area. The latter measurement may guide TV intervention for lesser degrees of TR when left sided surgery is performed. From a practical point, TS is usually identified in mixed TV disease or in association with MS.

\section{Therapy}

Once a diagnosis of ARF has been made definitive therapy to eradicate Strep A in the pharynx with penicillin is required. This can be either an intramuscular dose of benzathine penicillin or oral penicillin for 10 days. In cases where the diagnosis of ARF and definite RHD are made, intramuscular or oral penicillin is advocated as secondary prevention. This strategy reduces the risk of recurrent ARF and may diminish the progression of RHD (32-34). The duration of secondary prevention varies based on the age of the patient, the absence or presence of carditis, the last documented episode of ARF and whether persistent valvular damage is present. In patients with borderline RHD detected on screening echocardiography, 
secondary prevention is not routinely advocated since the natural history of these lesions are unpredictable and thus therapy has not proved to be clearly beneficial (35).

In patients with chronic RHD, prophylaxis for infective endocarditis is advocated by countries with endemic rates of RHD (36). This rationale is based on the poor prognosis of infective endocarditis in RHD that has been observed in some studies, the presence of poor dental hygiene in disadvantaged communities, and the possibility of coexisting immune deficiency.

\section{Heart failure and AF in patients with $R H D$}

In patients who present with heart failure, the use of diuretics, ACE inhibitors, digoxin and beta blockers are used to stabilize patients prior to definitive corrective surgery or as definitive therapy when surgery is not available, or patients are deemed surgically inoperable. The evidence for the utilization of these medical therapies has not been systematically studied but individualization of the therapeutic regime is based on the dominant hemodynamic valvular lesions, left ventricular function and clinical manifestations.

In patients with RHVD and AF, restoration of sinus rhythm maybe beneficial for selected patients with either amiodarone, cardioversion or even catheter ablation although definitive surgical correction of the primary valve lesions often offers the greatest predictable success. In most patients, rate control with digoxin or beta blockers are more widely used to improve symptoms especially when heart failure or significant MS is present. Prevention of cardioembolism, in particular stroke prevention is most commonly achieved with the use of vitamin $\mathrm{K}$ antagonists such as warfarin. Recently certain guidelines advocate the use of novel anticoagulants in patients with RHD with the exception of patients with MS, prosthetic valve replacements (37), or in the pregnant patient who requires anticoagulation. This therapeutic approach is based on limited observational data and will be more definitively answered in the INVestIgation of rheumatiC AF Treatment Using vitamin $\mathrm{K}$ antagonists, rivaroxaban or aspirin Studies (INVICTUS) trial that is currently being conducted. This trial will provide more evidence-based data on the safety and efficacy of direct oral anticoagulants (DOACs) versus vitamin $\mathrm{K}$ antagonists for the treatment of patients with RHD and AF in the near future. This trial also presents one of the first randomized controlled trials (RCTs) involving patients with RHD, generally a patient group not included in major trials. As new heart failure agents are being tested in RCTs, we urge investigators to consider this group of patients and to anticipate the issues with delivery of these medications.

\section{High risk groups-RHD in pregnancy}

Pregnancy represents a high-risk situation for women with RHD, especially those with obstructive left-sided valve lesions. A recent study from Uganda demonstrated significant maternal, neonatal and fetal risks in the context of all grades of RHD (38) while a previous study documented extremely poor outcomes for women with rheumatic MS (39).

The recent ESC guidelines for cardiovascular disease in pregnancy acknowledged the significant role of RHD in cardiac disease morbidity and mortality in pregnant women in endemic countries (40), although even more detailed management guidelines are needed in these regions (41). Special considerations include the timing of interventions or even surgery during pregnancy (largely thought safest in the mid-trimester), the use of Clexane versus Warfarin in those with prosthetic valves and most importantly the appropriate management and follow-up throughout pregnancy, the peri-partum period and to at least six months post-partum. We therefore advocate for careful pre-conceptual counselling in this population, with early discussion regarding reproductive planning and if possible, management in a combined cardio-obstetric team (42).

Both interventional and surgical management of RHD in children and women of reproductive age should be carefully considered and performed timeously. We advocate valve repairs and percutaneous interventions rather than replacements as far as possible (43), with a special emphasis again on pre-conceptual counselling, to ensure that future pregnancies are well planned (44).

\section{Invasive assessment and interventional management}

Echocardiography, in general, provides all the information needed for optimal management of patients with RHD. Invasive evaluation may be required when there is a discrepancy between echocardiographic findings and clinical symptoms, or as part of planned catheter intervention. Catheterization provides accurate estimates of left atrial pressures (measured as pulmonary capillary wedge pressure), ventricular filling pressures, and cardiac output, all of which can supplement information obtained from non-invasive testing and assist in formulating a therapeutic plan.

Patients with severe MS with suitable valve morphology, benefit most from catheter interventions. Consequently, percutaneous transvenous mitral commissurotomy (PTMC) is the most commonly performed intervention in patients 
with RHD. Though there have been improvements in hardware over the years, the basic procedure of PTMC is relatively unchanged from its original description by Kanji Inoue over 3 decades ago (45). The pivotal studies which established the percutaneous approach to the treatment of MS used single or double, conventional valvuloplasty balloons $(46,47)$. But the elegance and ease of use of the self-centering Inoue balloon has trumped these in clinical practice. Subsequent trials comparing PTMC with surgical approaches used the Inoue balloon and technique (48). Success of PTMC depends to a great extent on the morphology of the mitral valve. The presence of subvalvular fusion and calcification reduce the chances of a durable outcome. Several echocardiographic scores (49), and more complex multifactorial scores which use a combination of demographic, clinical and echocardiographic variables (50), are used to assess suitability for PTMC. Patients with MS are younger in RHD endemic developing countries and may have a lower prevalence of age-related morphological changes such as calcification and may therefore be more suitable candidates for PTMC. On the contrary, RHD may follow a more aggressive course in endemic countries resulting in severe morphologic abnormalities including subvalvular disease (51). Nevertheless, PTMC results in acceptable immediate and medium term outcomes, and remains the initial treatment of choice in these and most subsets of patients with other adverse demographic or clinical features $(52,53)$. The main complications associated with the procedure are severe MR needing urgent surgery (1-3\%), cardiac tamponade (1-2\%), systemic embolism $(<1 \%)$ and death $(<1 \%)(53)$. A meta-analysis of the small randomized studies comparing PTMC with surgical commissurotomy suggests that, compared to surgical commissurotomy, PTMC produces a slightly smaller valve area, a higher risk of $M R$, and a nearly three-fold risk of reintervention (54). Nevertheless, because of increasing familiarity, ease of use of the procedure, improvement in operator experience, and perhaps the lower direct and opportunity costs compared to surgical treatment, PTMC (using an Inoue or Inoue-like balloon) remains the treatment of choice for rheumatic MS.

The interventional treatment of rheumatic AS has not been studied as well, perhaps because of the rarity of isolated AS in RHD, and its tendency to manifest later in life (14), when valve calcification is common. But there is good rationale for the use of balloon dilatation to treat non-calcific rheumatic AS. In vitro studies have shown that balloon dilatation reliably splits the fused commissures in a rheumatic aortic valve (55). Data from a retrospective study suggest that balloon dilatation is associated with a high $(86 \%)$ immediate success rate, with only $14 \%$ of patients needing valve replacement at 5-year follow-up (56). However, moderate or severe AR can occur in about $14 \%$ of patients as an immediate complication of the procedure. Transcatheter aortic valve replacement is unlikely to be useful in RHD due to the rarity of isolated AS and the relatively younger age of patients with RHD.

Rheumatic TS is rare and occurs almost always in association with mitral valve disease, particularly MS. Limited data from small case series suggest that tricuspid valvuloplasty may be as successful and durable as PTMC $(57,58)$. A large-sized Inoue balloon (28 or $30 \mathrm{~mm}$ ) is usually used for dilating the TV.

MR is unlikely to be amenable to the transcatheter techniques used for non-rheumatic disease because of the presence of valve thickening, variable degrees of commissural fusion and subvalvular disease. Transcatheter treatment of severe TR may be more promising (59).

\section{Surgery for RHD}

The need for surgery for severe RHVD is clear with a significant lack of access to surgical and percutaneous intervention in regions of the greatest global need (60-62). Surgery for RHVD should be timeous to result in the best possible outcomes. However, in regions of the world where only one operation per patient is rationed, a valve replacement is often chosen above valve repair, despite the consequences for mechanical valves in countries without INR monitoring or regular warfarin supplies. Recently a thorough needs assessment has been suggested to evaluate cardiac surgery resources and inform practices in low and middle-income settings (63). Of importance is the growing understanding of SouthSouth cooperation and the need for local capacity building in creating comprehensive surgical programs in Africa (64) as well as surgical expertise to the health equity agenda (65).

\section{Conclusions}

RHD remains a neglected cardiovascular disease which causes significant morbidity and mortality in low- and middleincome countries. Although the recent Jones criteria update has been designed to consider the important differences in epidemiology, diagnostic capacity is hampered by human and technological resource scarcity in RHD-endemic countries and regions. This is most pronounced in the need for cardiac surgery which is not available in most parts of the world with the highest RHD burden. We advocate for RHD as a global health priority, as the only preventable cardiovascular disease, 
and for efforts to increase the treatment armamentarium as well as advocacy, funding and research.

\section{Acknowledgments}

L Zühlke and J Abrams gratefully acknowledges funding from Medtronic foundation through funding to RHDAction, L Zühlke is also funded through the Medical Research Council of South Africa, the National Research Foundation of South Africa and MRCUK Dfid through the African Research Leader Scheme.

Funding: None.

\section{Footnote}

Provenance and Peer Review: This article was commissioned by the Guest Editor (Ntobeko A. B. Ntusi) for the series "Cardiovascular Diseases in Low-and Middle-Income Countries" published in Cardiovascular Diagnosis and Therapy. The article was sent for external peer review organized by the Guest Editor and the editorial office.

Conflicts of Interest: All authors have completed the ICMJE uniform disclosure form (available at http://dx.doi. org/10.21037/cdt.2019.10.07). The series "Cardiovascular Diseases in Low- and Middle-Income Countries" was commissioned by the editorial office without any funding or sponsorship. The authors have no other conflicts of interest to declare.

Ethical Statement: The authors are accountable for all aspects of the work in ensuring that questions related to the accuracy or integrity of any part of the work are appropriately investigated and resolved.

Open Access Statement: This is an Open Access article distributed in accordance with the Creative Commons Attribution-NonCommercial-NoDerivs 4.0 International License (CC BY-NC-ND 4.0), which permits the noncommercial replication and distribution of the article with the strict proviso that no changes or edits are made and the original work is properly cited (including links to both the formal publication through the relevant DOI and the license). See: https://creativecommons.org/licenses/by-nc-nd/4.0/.

\section{References}

1. He VY, Condon JR, Ralph AP, et al. Long-Term Outcomes
From Acute Rheumatic Fever and Rheumatic Heart Disease: A Data-Linkage and Survival Analysis Approach. Circulation 2016;134:222-32.

2. Okello E, Kakande B, Sebatta E, et al. Socioeconomic and environmental risk factors among rheumatic heart disease patients in Uganda. PLoS One 2012;7:e43917.

3. Watkins DA, Beaton AZ, Carapetis JR, et al. Rheumatic Heart Disease Worldwide: JACC Scientific Expert Panel. J Am Coll Cardiol 2018;72:1397-416.

4. Cannon J, Roberts K, Milne C, et al. Rheumatic Heart Disease Severity, Progression and Outcomes: A MultiState Model. J Am Heart Assoc 2017. doi: 10.1161/ JAHA.116.003498.

5. Remenyi B, Carapetis J, Wyber R, et al. Position statement of the World Heart Federation on the prevention and control of rheumatic heart disease. Nat Rev Cardiol 2013;10:284-92 .

6. Yacoub M, Mayosi B, ElGuindy A, et al. Eliminating acute rheumatic fever and rheumatic heart disease. Lancet 2017;390:212-3.

7. Sliwa K, White A, Milan P, et al. Momentum builds for a global response to rheumatic heart disease. Eur Heart J 2018;39:4229-32.

8. Watkins DA, Johnson CO, Colquhoun SM, et al. Global, Regional, and National Burden of Rheumatic Heart Disease, 1990-2015. N Engl J Med 2017;377:713-22.

9. Gewitz MH, Baltimore RS, Tani LY, et al. Revision of the Jones Criteria for the diagnosis of acute rheumatic fever in the era of Doppler echocardiography: a scientific statement from the American Heart Association. Circulation 2015;131:1806-18.

10. Zaman MM, Hassan MM, Ahmed J, et al. Streptococcal antibodies among rural school children in Bangladesh. Bangladesh Med Res Counc Bull 2002;28:1-6.

11. Roberts K, Colquhoun S, Steer A, et al. Screening for rheumatic heart disease: current approaches and controversies. Nat Rev Cardiol 2013;10:49-58.

12. Minich LL, Tani LY, Pagotto LT, et al. Doppler echocardiography distinguishes between physiologic and pathologic "silent" mitral regurgitation in patients with rheumatic fever. Clin Cardiol 1997;20:924-6.

13. Karthikeyan G, Guilherme L. Acute rheumatic fever. Lancet 2018;392:161-74.

14. Zühlke L, Engel ME, Karthikeyan G, et al. Characteristics, complications, and gaps in evidence-based interventions in rheumatic heart disease: the Global Rheumatic Heart Disease Registry (the REMEDY study). Eur Heart J 2015;36:1115-22a.

15. Okello E, Wanzhu Z, Musoke C, et al. Cardiovascular 
complications in newly diagnosed rheumatic heart disease patients at Mulago Hospital, Uganda. Cardiovasc J Afr 2013;24:80-5.

16. Ali S, Domi S, Abbo B, et al. Echocardiographic screening for rheumatic heart disease in 4515 Sudanese school children: marked disparity between two communities. Cardiovasc J Afr 2018;29:273-7.

17. Marijon E, Celermajer DS, Tafflet M, et al. Rheumatic heart disease screening by echocardiography: the inadequacy of World Health Organization criteria for optimizing the diagnosis of subclinical disease. Circulation 2009; 120:663-8.

18. Marijon E, Ou P, Celermajer DS, et al. Prevalence of rheumatic heart disease detected by echocardiographic screening. N Engl J Med 2007;357:470-6.

19. Mirabel M, Fauchier T, Bacquelin R, et al.

Echocardiography screening to detect rheumatic heart disease: A cohort study of schoolchildren in French Pacific Islands. Int J Cardiol 2015;188:89-95.

20. Reményi B, Wilson N, Steer A, et al. World Heart Federation criteria for echocardiographic diagnosis of rheumatic heart disease--an evidence-based guideline. Nat Rev Cardiol 2012;9:297-309.

21. Musuku J, Engel ME, Musonda P, et al. Prevalence of rheumatic heart disease in Zambian school children. BMC Cardiovasc Disord 2018;18:135.

22. Yadeta D, Hailu A, Haileamlak A, et al. Prevalence of rheumatic heart disease among school children in Ethiopia: A multisite echocardiography-based screening. Int J Cardiol 2016;221:260-3.

23. Sims Sanyahumbi A, Sable CA, Beaton A, et al. School and Community Screening Shows Malawi, Africa, to Have a High Prevalence of Latent Rheumatic Heart Disease. Congenit Heart Dis 2016;11:615-21.

24. Sanyahumbi A, Beaton A, Guffey D, et al. Two-year evolution of latent rheumatic heart disease in Malawi. Congenit Heart Dis 2019;14:614-8.

25. Sliwa K, Carrington M, Mayosi BM, et al. Incidence and characteristics of newly diagnosed rheumatic heart disease in urban African adults: insights from the heart of Soweto study. Eur Heart J 2010;31:719-27.

26. Meel R, Peters F, Libhaber E, et al. The changing spectrum of rheumatic mitral regurgitation in Soweto, South Africa. Cardiovasc J Afr 2017;28:215-20.

27. Anthony J, Osman A, Sani MU. Valvular heart disease in pregnancy. Cardiovasc J Afr 2016;27:111-8.

28. van Hagen IM, Thorne SA, Taha N, et al. Pregnancy Outcomes in Women With Rheumatic Mitral Valve
Disease: Results From the Registry of Pregnancy and Cardiac Disease. Circulation 2018;137:806-16.

29. Roos-Hesselink J, Baris L, Johnson M, et al. Pregnancy outcomes in women with cardiovascular disease: evolving trends over 10 years in the ESC Registry Of Pregnancy And Cardiac disease (ROPAC). Eur Heart J 2019;40:3848-55.

30. Anthony J, Sliwa K. Decompensated Heart Failure in Pregnancy. Card Fail Rev 2016;2:20-6.

31. Zühlke L, Karthikeyan G, Engel ME, et al. Clinical Outcomes in 3343 Children and Adults With Rheumatic Heart Disease From 14 Low- and Middle-Income Countries: Two-Year Follow-Up of the Global Rheumatic Heart Disease Registry (the REMEDY Study). Circulation 2016;134:1456-66.

32. Gerber MA, Baltimore RS, Eaton CB, et al. Prevention of rheumatic fever and diagnosis and treatment of acute Streptococcal pharyngitis: a scientific statement from the American Heart Association Rheumatic Fever, Endocarditis, and Kawasaki Disease Committee of the Council on Cardiovascular Disease in the Young, the Interdisciplinary Council on Functional Genomics and Translational Biology, and the Interdisciplinary Council on Quality of Care and Outcomes Research: endorsed by the American Academy of Pediatrics. Circulation 2009;119:1541-51.

33. Manyemba J, Mayosi BM. Penicillin for secondary prevention of rheumatic fever. Cochrane Database Syst Rev 2002;(3):CD002227.

34. Manyemba J, Mayosi BM. Intramuscular penicillin is more effective than oral penicillin in secondary prevention of rheumatic fever--a systematic review. S Afr Med J 2003;93:212-8.

35. Fernandes JR, Grinberg M. Prophylaxis of infective endocarditis: a different Brazilian reality? Arq Bras Cardiol 2013;101:e37-8.

36. Jankelow D, Cupido BJ, Zühlke LJ, et al. Prevention of infective endocarditis associated with dental interventions. SA Heart 2017;14:170-4.

37. Nishimura RA, Otto CM, Bonow RO, et al. 2014 AHA/ ACC Guideline for the Management of Patients With Valvular Heart Disease: A Report of the American College of Cardiology/American Heart Association Task Force on Practice Guidelines. Circulation 2014;129:e521-643.

38. Beaton A, Okello E, Scheel A, et al. Impact of heart disease on maternal, fetal and neonatal outcomes in a low-resource setting. Heart 2019;105:755-60.

39. Diao M, Kane A, Ndiaye MB, et al. Pregnancy in women with heart disease in sub-Saharan Africa. Arch Cardiovasc 
Dis 2011;104:370-4.

40. Regitz-Zagrosek V, Roos-Hesselink JW, Bauersachs J, et al. 2018 ESC Guidelines for the management of cardiovascular diseases during pregnancy. Eur Heart J 2018;39:3165-241.

41. Mocumbi AO, Jamal KK, Mbakwem A, et al. The Pan-African Society of Cardiology position paper on reproductive healthcare for women with rheumatic heart disease. Cardiovasc J Afr 2018;29:394-403.

42. Sliwa K, Azibani F, Baard J, et al. Reducing late maternal death due to cardiovascular disease - A pragmatic pilot study. Int J Cardiol 2018;272:70-6.

43. Remenyi B, Webb R, Gentles T, et al. Improved longterm survival for rheumatic mitral valve repair compared to replacement in the young. World J Pediatr Congenit Heart Surg 2013;4:155-64.

44. Vaughan G, Tune K, Peek MJ, et al. Rheumatic heart disease in pregnancy: strategies and lessons learnt implementing a population-based study in Australia. Int Health 2018;10:480-9.

45. Inoue $\mathrm{K}$, Owaki T, Nakamura $\mathrm{T}$, et al. Clinical application of transvenous mitral commissurotomy by a new balloon catheter. J Thorac Cardiovasc Surg 1984;87:394-402.

46. Shrivastava S, Mathur A, Dev V, et al. Comparison of immediate hemodynamic response to closed mitral commissurotomy, single-balloon, and double-balloon mitral valvuloplasty in rheumatic mitral stenosis. J Thorac Cardiovasc Surg 1992;104:1264-7.

47. Lock JE, Khalilullah M, Shrivastava S, et al. Percutaneous catheter commissurotomy in rheumatic mitral stenosis. $\mathrm{N}$ Engl J Med 1985;313:1515-8.

48. Reyes VP, Raju BS, Wynne J, et al. Percutaneous balloon valvuloplasty compared with open surgical commissurotomy for mitral stenosis. N Engl J Med 1994;331:961-7.

49. Wilkins GT, Weyman AE, Abascal VM, et al. Percutaneous balloon dilatation of the mitral valve: an analysis of echocardiographic variables related to outcome and the mechanism of dilatation. Br Heart J 1988;60:299-308.

50. Palacios IF, Sanchez PL, Harrell LC, et al. Which patients benefit from percutaneous mitral balloon valvuloplasty? Prevalvuloplasty and postvalvuloplasty variables that predict long-term outcome. Circulation 2002;105:1465-71.

51. Roy SB, Bhatia ML, Lazaro EJ, et al. Juvenile Mitral Stenosis in India. Lancet 1963;2:1193-5.

52. Bahl VK, Chandra S, Talwar KK, et al. Influence of subvalvular fibrosis on results and complications of percutaneous mitral commissurotomy with use of the Inoue balloon. Am Heart J 1994;127:1554-8.
53. Palacios IF, Arzamendi D. Percutaneous Mitral Balloon Valvuloplasty for Patients with Rheumatic Mitral Stenosis. Interv Cardiol Clin 2012;1:45-61.

54. Hu X, Zhao Q. Systematic comparison of the effectiveness of percutaneous mitral balloon valvotomy with surgical mitral commissurotomy. Swiss Med Wkly 2011;141:w13180.

55. Ribeiro PA, Al Zaibag M, Rajendran V. Double balloon aortic valvotomy for rheumatic aortic stenosis; in vivo studies. Eur Heart J 1989;10:417-22.

56. Pillai AA, Ramasamy C, Saktheeshwaran M, et al. Balloon valvuloplasty in rheumatic aortic valve stenosis: immediate and long-term results. Cardiovasc Interv Ther 2015;30:45-50.

57. Al Zaibag M, Ribeiro P, Al Kasab S. Percutaneous balloon valvotomy in tricuspid stenosis. British Heart Journal 1987;57:51-3.

58. Sancaktar O, Kumbasar SD, Semiz E, et al. Late results of combined percutaneous balloon valvuloplasty of mitral and tricuspid valves. Cathet Cardiovasc Diagn 1998;45:246-50.

59. Van Praet KM, Stamm C, Starck CT, et al. An overview of surgical treatment modalities and emerging transcatheter interventions in the management of tricuspid valve regurgitation. Expert Rev Cardiovasc Ther 2018;16:75-89.

60. Zilla P, Bolman RM, Yacoub MH, et al. The Cape Town Declaration on Access to Cardiac Surgery in the Developing World. Ann Thorac Surg 2018;106:930-3.

61. Zilla P, Yacoub M, Zuhlke L, et al. Global Unmet Needs in Cardiac Surgery. Glob Heart 2018;13:293-303.

62. Zilla P, Zuhlke L, Sliwa K, et al. The African context of the Cape Town Declaration. Cardiovasc J Afr 2018;29:204.

63. Forcillo J, Watkins DA, Brooks A, et al. Making cardiac surgery feasible in African countries: Experience from Namibia, Uganda, and Zambia. J Thorac Cardiovasc Surg 2019;158:1384-93.

64. Shidhika FF, Hugo-Hamman CT, Lawrenson JB, et al. The Namibian Children's Heart Project: a SouthSouth partnership to provide cardiac care. Cardiol Young 2019;29:206-13.

65. Binagwaho A, Muhimpundu MA, Bukhman G, et al. 80 under 40 by 2020: an equity agenda for NCDs and injuries. Lancet 2014;383:3-4.

Cite this article as: Peters F, Karthikeyan G, Abrams J, Muhwava L, Zühlke L. Rheumatic heart disease: current status of diagnosis and therapy. Cardiovasc Diagn Ther 2020;10(2):305315. doi: 10.21037/cdt.2019.10.07 